\title{
DYNAMICS OF A GALACTIC HAMILTONIAN SYSTEM
}

\author{
ERnesto A. LACOMba \\ Departamento de Matemáticas, Universidad Autónoma Metropolitana, \\ Iztapalapa, Av. San Rafael Atlixco 186, 09340, Mexico, D.F. \\ e-mail: lace@xanum.uam.mx \\ JAUME LLIBRE \\ Departament de Matemàtiques, Universitat Autònoma de Barcelona, \\ 08193 Bellaterra, Barcelona, Catalonia, Spain \\ e-mail: jllibre@mat.uab.cat \\ This is the corresponding author. \\ Tel. +34 935811303. Fax +34 935812790. ${ }^{1}$
}

\begin{abstract}
We study an even polynomial potential which appears in the study of the galactic dynamics. We prove the existence of four families of periodic orbits in every positive energy level, and we compute an analytic approximation of them. Using such periodic orbits we provide information about the non-integrability of this Hamiltonian system.
\end{abstract}

\footnotetext{
${ }^{1} 2010$ Mathematics Subject Classification. Primary 34C10, 34C25.

Key words and phrases. periodic orbits, integrability, galactic potential, averaging theory.
} 


\section{IntroduCtion AND STATEMENTS OF MAIN RESUlTS}

To determine interesting properties of the orbital structure of non-integrable potentials is a fundamental topic in galactic dynamics. To know the existence and stability of periodic orbits of low commensurability is important for clarifying some behaviors of the elliptical galaxies. The numerical studies are usually preferred due to the availability of reliable algorithms and powerful machines. On the other hand, in several cases it is useful to have some simple analytic results concerning the relation between the form of the gravitational potential and the main families of orbits exhibited.

We consider two degrees of freedom natural Hamiltonian systems of the form

$$
H=H\left(q_{1}, q_{2}, p_{1}, p_{2}\right)=\frac{1}{2}\left(p_{1}^{2}+p_{2}^{2}\right)+V\left(q_{1}^{2}, q_{2}^{2}\right),
$$

with $V$ a smooth potential with an absolute minimum and reflection symmetry with respect to both axes, see for more details [17]. The reason for the choice of these symmetric systems is due to their interest in problems of galactic dynamics. More precisely, we will examine the following potential

$$
V=V\left(q_{1}, q_{2}\right)=\frac{1}{2}\left(q_{1}^{2}+\frac{q_{2}^{2}}{k}\right)+\varepsilon\left(a q_{1}^{4}+b q_{1}^{2} q_{2}^{2}+c q_{2}^{4}\right),
$$

for the case $k=1$.

The first objective of this paper is to study analytically the periodic orbits of the two degrees of freedom Hamiltonian system (2) having $k=1$, while the Hamiltonian for the case $k$ irrational is being studied in [2]. More precisely, we shall see that the Weinstein's Theorem (stated in the appendix) shows the existence of four families of periodic orbits bifurcating from the periodic orbits of the linear differential system associated to the Hamiltonian one. We shall use the averaging theory for computing an explicit analytic approximation of these four families of periodic orbits for $\varepsilon>0$ sufficiently small. Additionally the averaging method allows one to study the stability or instability of these periodic orbits.

The Hamiltonian system to be studied here is

$$
\begin{aligned}
& \dot{q}_{1}=p_{1}, \\
& \dot{q}_{2}=p_{2}, \\
& \dot{p}_{1}=-q_{1}-\varepsilon\left(4 a q_{1}^{3}+2 b q_{1} q_{2}^{2}\right), \\
& \dot{p}_{2}=-q_{2}-\varepsilon\left(2 b q_{1}^{2} q_{2}+4 c q_{2}^{3}\right) .
\end{aligned}
$$

As usual the dot denotes derivative with respect to the independent variable $t$, the time.

The periodic orbits are the most simple non-trivial solutions of a differential system. Their study is of particular interest because the motion in their neighborhood can be determined by their kind of stability. Furthermore, the 
existence of isolated periodic orbits in the energy levels of a Hamiltonian system with multipliers different from 1 forces, under convenient assumptions, the non-existence of any second first integral of class $\mathcal{C}^{1}$. So the study of the periodic orbits for a differential system becomes relevant for several reasons. All the notions mentioned in this paragraph will be defined later on.

In this work we use the averaging method of first order to compute periodic orbits as it is established in $[18,5]$, see the appendix for a summary of this method. This method allows to find periodic orbits of our Hamiltonian system (3), up to first order in $\varepsilon$, at any Hamiltonian level $H=h>0$ as a function of the parameter $h$. Roughly speaking, this method reduces the problem of finding periodic solutions of some differential system to the one of finding zeros of some convenient finite dimensional function. In $[7,8]$ the application of this technique has been considered in order to obtain periodic solutions of some well known Hamiltonian systems.

Our main result about the periodic orbits of the Hamiltonian system (3) is summarized as follows, where $r=\sqrt{q_{1}^{2}+p_{1}^{2}}$ and $\rho=\sqrt{q_{2}^{2}+p_{2}^{2}}$.

Theorem 1. For $\varepsilon>0$ sufficiently small in every energy level $H=h>0$ the perturbed Hamiltonian system (3) may have 4 periodic solutions bifurcating from the periodic orbits of the unperturbed Hamiltonian system, as follows

(a) The first one comes from the periodic orbit $r=0$ and $\rho=\sqrt{2 h}$ of system (3) with $\varepsilon=0$ if $(b-6 c)(b-2 c)$ is nonzero.

(b) The second one comes from the periodic orbit $r=\sqrt{2 h}$ and $\rho=0$ of system (3) with $\varepsilon=0$ if $(6 a-b)(2 a-b)$ is nonzero.

(c) The third one comes from the periodic orbit $r=\sqrt{\frac{(6 c-b) h}{3 a-b-3 c}}$ and $\rho=$ $\sqrt{\frac{(6 a-b) h}{3 a-b-3 c}}$ of system (3) with $\varepsilon=0$ if $b(6 a-b)(6 c-b)(3 a-b+3 c)$ is nonzero.

(d) The fourth one comes from the periodic orbit $r=\sqrt{\frac{(2 c-b) h}{a-b+c}}$ and $\rho=\sqrt{\frac{(2 a-b) h}{a-b+c}}$ of system (3) with $\varepsilon=0$ if $b(2 a-b)(b-2 c)(a-b+c)$ is nonzero.

Theorem 1 is proved in Section 2 using the averaging theory.

It is well known that integrable and non-integrable Hamiltonian systems can have infinitely many periodic orbits. However in general it is not easy to prove the existence of families of periodic orbits in an analytical way, specially if the Hamiltonian system is non-integrable. We can use these families of periodic orbits to prove our second main result about the $\mathcal{C}^{1}$ non-integrability in the sense of Liouville-Arnol'd of Hamiltonian system (3) with polynomial potential. See the appendix for a precise definition of a Liouville-Arnol'd integrable Hamiltonian system. 
Theorem 2. The Hamiltonian system (3) with Hamiltonian $H$ cannot have $a \mathcal{C}^{1}$ second first integral $G$ such that the gradients of $H$ and $G$ are linearly independent at each point of the periodic orbits found in Theorem 1.

Theorem 2 is proved in section 3 .

Our study on the non Liouville-Arnol'd integrability uses isolated periodic orbits in the Hamiltonian levels, but other studies as the Moser-Holmes proof (see $[15,6]$ ) use transverse homoclinic orbits. It is important to remark that both our method and Moser-Holmes method work for Hamiltonian systems which are close to integrable systems.

Another interesting criterion on non-integrability also related with the Poincaré's result was used by Meletlidou and Ichtiarouglou [11, 12, 13]. They consider perturbed Hamiltonian systems of the form $H=H_{0}+\varepsilon H_{1}$, where $H_{0}$ is a non-degenerate integrable Hamiltonian, and they show that some properties of the averaged value of the perturbing function $H_{1}$, evaluated along the non-isolated periodic orbits of $H_{0}$, are strongly connected with the non-integrability of the perturbed system. This criterion cannot be applied to the Hamiltonian system (3) due to its degeneracy.

\section{Proof of Theorem 1}

Probably the more general theorem for studying the periodic orbits which are born from the linear part of Hamiltonian system is the Weinstein's Theorem, stated in the appendix. This theorem needs that the Hessian matrix $H_{x x}(O)$ with $x=\left(q_{1}, q_{2}, p_{1}, p_{2}\right)$, of the Hamiltonian function (1) with the potential (2) at the equilibrium point $O=(0,0,0,0)$ of the Hamiltonian system (3), be positive definite, which is the case because $H_{x x}(O)=I d$. Then, the Weinstein's Theorem states for our Hamiltonian system (3) the existence of four families of periodic orbits which are born from the periodic orbits of the linear part of the Hamiltonian system at the origin, but does not provide an analytic approximation of these periodic orbits. Now using the averaging theory method we shall provide an explicit analytic approximation of these four families of periodic orbits, and in addition we shall obtain information on their stability.

Proof of Theorem 1. For proving Theorem 1 we shall apply Theorem 5 of the appendix to the Hamiltonian system (3). Generically the periodic orbits of a Hamiltonian system with more than one degree of freedom live on cylinders filled by periodic orbits. Therefore we cannot apply directly Theorem 5 to a Hamiltonian system because the averaging method stated in Theorem 5 only detects isolated periodic orbits in the set of all periodic orbits. Therefore we must apply Theorem 5 to every fixed Hamiltonian level where the periodic orbits are generically isolated. Remember that the integrable Hamiltonian systems in the sense of Liouville-Arnol'd are non-generic, see [10].

Moreover system (3) for $\varepsilon$ sufficiently small is close to the integrable one for $\varepsilon=0$. 
In order to prepare the application of the averaging method described in the appendix, we begin by changing the first integral (1) and the equations of motion (3) to convenient generalized polar coordinates in such a way that for $\varepsilon=0$ we have a pair of harmonic oscillators whose solution is

$$
\begin{array}{ll}
q_{1}(t)=q_{10} \cos t+p_{10} \sin t, & p_{1}(t)=-q_{10} \sin t+p_{10} \cos t, \\
q_{2}(t)=q_{20} \cos t+p_{10} \sin t, & p_{2}(t)=-q_{20} \sin t+p_{20} \cos t,
\end{array}
$$

and with energy $h=\frac{1}{2}\left(p_{10}^{2}+p_{20}^{2}+q_{10}^{2}+q_{20}^{2}\right)$. Thus we consider the change of variables $\left(q_{1}, q_{2}, p_{1}, p_{2}\right) \rightarrow(r, \theta, \rho, \alpha) \in \mathbb{R}^{+} \times \mathbb{S}^{1} \times \mathbb{R}^{+} \times \mathbb{S}^{1}$ given by

$$
q_{1}=r \cos \theta, \quad p_{1}=r \sin \theta, \quad q_{2}=\rho \cos (\theta+\alpha), \quad p_{2}=\rho \sin (\theta+\alpha) .
$$

Moreover doing this change of variables, the angular variables $\theta$ and $\alpha$ appear in the system. Later on the variable $\theta$ will be used for obtaining the periodicity necessary for applying the averaging theory.

In short in the new variables the first integral is

$\mathcal{H}=\frac{1}{2}\left(r^{2}+\rho^{2}\right)+\varepsilon\left[a r^{4} \cos ^{4} \theta+b r^{2} \rho^{2} \cos ^{2} \theta \cos ^{2}(\theta+\alpha)+c \rho^{4} \cos ^{4}(\theta+\alpha)\right]$,

and the equations of motion are

(6)

$$
\begin{aligned}
\dot{r} & =-\varepsilon 2 r \cos \theta \sin \theta\left[2 a r^{2} \cos ^{2} \theta+b \rho^{2} \cos ^{2}(\theta+\alpha)\right], \\
\dot{\rho} & =-\varepsilon 2 \rho \cos (\theta+\alpha) \sin (\theta+\alpha)\left[b r^{2} \cos ^{2} \theta+2 c \rho^{2} \cos ^{2}(\theta+\alpha)\right], \\
\dot{\theta} & =-1-\varepsilon 2 \cos ^{2} \theta\left[2 a r^{2} \cos ^{2} \theta+b \rho^{2} \cos ^{2}(\theta+\alpha)\right], \\
\dot{\alpha} & =\varepsilon\left[4 a r^{2} \cos ^{4} \theta+2 b\left(\rho^{2}-r^{2}\right) \cos ^{2} \theta \cos ^{2}(\theta+\alpha)-4 c \rho^{2} \cos ^{4}(\theta+\alpha)\right] .
\end{aligned}
$$

Remember that the derivatives of the left hand side of these equations are with respect to the time variable $t$, which is not periodic. The angular variable $\alpha$ cannot be used as the independent variable because the new differential system would not have the form (14) for applying Theorem 5. As for $\varepsilon>0$ sufficiently small we have $\dot{\theta}<0$, we can change to the $\theta$ variable as the independent one, and we denote by a prime the derivative with respect to $\theta$, getting the system

(7)

$$
\begin{aligned}
r^{\prime} & =\varepsilon 2 r \cos \theta \sin \theta\left[2 a r^{2} \cos ^{2} \theta+b \rho^{2} \cos ^{2}(\theta+\alpha)\right]+O\left(\varepsilon^{2}\right), \\
\rho^{\prime} & =\varepsilon 2 \rho \cos (\theta+\alpha) \sin (\theta+\alpha)\left[b r^{2} \cos ^{2} \theta+2 c \rho^{2} \cos ^{2}(\theta+\alpha)\right]+O\left(\varepsilon^{2}\right), \\
\alpha^{\prime} & =-\varepsilon\left[4 a r^{2} \cos ^{4} \theta+2 b\left(\rho^{2}-r^{2}\right) \cos ^{2} \theta \cos ^{2}(\theta+\alpha)-4 c \rho^{2} \cos ^{4}(\theta+\alpha)\right]+O\left(\varepsilon^{2}\right) .
\end{aligned}
$$

We shall study the motion of the differential system (7) at the level $\mathcal{H}=$ $h>0$ of its first integral $\mathcal{H}$ given by (5). Then we solve the equation $\mathcal{H}(r, \theta, \rho, \alpha)=h$ for $\rho$, and expanding $\rho$ in Taylor series to first order in $\varepsilon$ we obtain

$$
\rho=\sqrt{2 h-r^{2}}+O(\varepsilon)=\sqrt{p_{10}^{2}+p_{20}^{2}+q_{10}^{2}+q_{20}^{2}-r^{2}}+O(\varepsilon) .
$$


If we write the differential equations of $r^{\prime}$ and $\alpha^{\prime}$, substituting in them the expression of $\rho$ given by (8), and expanding them in Taylor series in powers of $\varepsilon$, we obtain the differential system

$$
\begin{aligned}
& r^{\prime}=\varepsilon F_{11}+O\left(\varepsilon^{2}\right), \\
& \alpha^{\prime}=\varepsilon F_{12}+O\left(\varepsilon^{2}\right) .
\end{aligned}
$$

where

$$
\begin{aligned}
F_{11}= & 2 r \cos \theta \sin \theta\left[2 a r^{2} \cos ^{2} \theta+b\left(p_{10}^{2}+p_{20}^{2}+q_{10}^{2}+q_{20}^{2}-r^{2}\right) \cos ^{2}(\theta+\alpha)\right] \\
& +O\left(\varepsilon^{2}\right), \\
F_{12}= & -\left[4 a r^{2} \cos ^{4} \theta+2 b\left(p_{10}^{2}+p_{20}^{2}+q_{10}^{2}+q_{20}^{2}-2 r^{2}\right) \cos ^{2} \theta \cos ^{2}(\theta+\alpha)\right. \\
& \left.-4 c\left(p_{10}^{2}+p_{20}^{2}+q_{10}^{2}+q_{20}^{2}-r^{2}\right) \cos ^{4}(\theta+\alpha)\right]+O\left(\varepsilon^{2}\right) .
\end{aligned}
$$

Clearly system (9) satisfies the assumptions of Theorem 5 , and it has the form (14) with the analytic function $F_{1}=\left(F_{11}, F_{12}\right)$. Furthermore this function is $2 \pi$-periodic in the variable $\theta$, the independent variable of system (9). In order to apply the averaging theory of first order we must calculate the averaged functions of $F_{11}$ and $F_{12}$ as follows

(10)

$$
\begin{aligned}
& f_{1}(r, \alpha)=\frac{1}{2 \pi} \int_{0}^{2 \pi} F_{11} d \theta=-\frac{1}{2} b r \cos \alpha \sin \alpha\left(2 h-r^{2}\right) \\
& f_{2}(r, \alpha)=\frac{1}{2 \pi} \int_{0}^{2 \pi} F_{12} d \theta=-\frac{3}{2} a r^{2}+\frac{3}{2} c\left(2 h-r^{2}\right)-\frac{1}{4} b\left(2 h-2 r^{2}\right)(2+\cos 2 \alpha) .
\end{aligned}
$$

We need to compute the real solutions $\left(r^{*}, \alpha^{*}\right)$ of $f_{1}(r, \alpha)=f_{2}(r, \alpha)=0$ such that the Jacobian

$$
\left|D_{r, \alpha}\left(f_{1}, f_{2}\right)\right|_{\left(r^{*}, \alpha^{*}\right)}
$$

is nonzero. We eliminate solutions which provide the same periodic orbit but with different initial conditions, or solutions with $r<0$, getting the following four possible solutions if we recall that $2 h=r^{2}+\rho^{2}$ when $\varepsilon=0$.

(a) The first one is the periodic orbit $r=0$ and $\alpha=-\frac{1}{2} \arccos \left(\frac{6 c}{b}-2\right)$, or equivalently $r=0$ and $\rho=\sqrt{2 h}$. This is a periodic orbit of the harmonic oscillator contained in the plane $\left(q_{1}, p_{1}\right)$.

(b) The second one is the periodic orbit $r=\sqrt{2 h}$ and $\alpha=-\frac{1}{2} \arccos \left(\frac{6 a}{b}-\right.$ 2 ), or equivalently $r=\sqrt{2 h}$ and $\rho=0$. This is a periodic orbit of the harmonic oscillator contained in the plane $\left(q_{2}, p_{2}\right)$.

(c) The third one is the periodic orbit $r=\sqrt{\frac{(6 c-b) h}{3 a-b+3 c}}$ and $\alpha=\pi / 2$, which implies $\rho=\sqrt{\frac{(6 a-b) h}{3 a-b-3 c}}$.

(d) The fourth one is the periodic orbit $r=\sqrt{\frac{(2 c-b) h}{a-b+c}}$ and $\alpha=0$, which implies $\rho=\sqrt{\frac{(2 a-b) h}{a-b+c}}$. 
In each one of these solutions the Jacobian (11) takes respectively the values

$$
\begin{aligned}
& \frac{3}{2}(b-6 c)(b-2 c) h^{2}, \\
& 3(b-6 a)(b-2 a) h^{2}, \\
& \frac{(b-6 a) b(b-6 c) h^{2}}{2(3 a-b+3 c)}, \\
& -\frac{3(b-2 a) b(b-2 c) h^{2}}{2(a-b+c)} .
\end{aligned}
$$

So by Theorem 5 , these are the possible four periodic solutions described in the statement of Theorem 1.

\section{Proof of Theorem 2}

We consider the four families of periodic orbits given in Theorem 1. Since the Jacobians (11) corresponding to the four periodic orbits depend on the 3 parameters $a, b, c$ and on the energy level $h$, they are in general different from 1, and these Jacobians are the product of two multipliers of these periodic orbits. Therefore, it follows that such two multipliers are in general different from 1 . For more details on the computations of the multipliers see the appendix. Hence, Theorem 2 follows from Theorem 4.

\section{Conclusions}

We have used two important tools in the area of dynamical systems. First the averaging theory for studying the existence of periodic orbits and their stability adapted to Hamiltonian systems. The main results on the periodic orbits of the Hamiltonian system (3) are summarized in Theorem 1. The second tool based in the Theorem 4 of Poincare allows to study the $\mathcal{C}^{1}$ nonintegrability in the sense of Liouville-Arnol'd of the Hamiltonian systems. Theorem 2 summarizes this result for our galactic potential.

\section{Appendix}

In this section we summarize some facts on the Liouville-Arnol'd integrability of the Hamiltonian systems, and on the theory of the periodic orbits of the differential equations, for more details see [1] and the subsection 7.1.2 of [3], respectively. We present these results for Hamiltonian systems of two degrees of freedom, because we are studying the Hamiltonian system (3) with two degrees of freedom, but these results work for an arbitrary number of degrees of freedom. 
5.1. Liouville-Arnol'd Theorem. We recall that a Hamiltonian system with Hamiltonian $H$ of two degrees of freedom is integrable in the sense of Liouville-Arnol'd if it has a first integral $C$ independent with $H$ (i.e. the gradient vectors of $H$ and $C$ are independent in all the points of the phase space except perhaps in a set of zero Lebesgue measure), and in involution with $H$ (i.e. the parenthesis of Poisson of $H$ and $C$ is zero). For Hamiltonian systems with two degrees of freedom the involution condition is redundant, because the fact that $C$ is a first integral of the Hamiltonian system, implies that the mentioned Poisson parenthesis is always zero. A flow defined on a subspace of the phase space is complete if its solutions are defined for all time.

Now we shall state the Liouville-Arnol'd Theorem restricted to Hamiltonian systems of two degrees of freedom.

Theorem 3. Suppose that a Hamiltonian system with two degrees of freedom defined on the phase space $M$ has its Hamiltonian $H$ and the function $C$ as two independent first integrals in involution. If $I_{h c}=\{p \in M: H(p)=$ $h$ and $C(p)=c\} \neq \emptyset$ and $(h, c)$ is a regular value of the map $(H, C)$, then the following statements hold.

(a) $I_{h c}$ is a two dimensional submanifold of $M$ invariant under the flow of the Hamiltonian system.

(b) If the flow on a connected component $I_{h c}^{*}$ of $I_{h c}$ is complete, then $I_{h c}^{*}$ is diffeomorphic either to the torus $\mathbb{S}^{1} \times \mathbb{S}^{1}$, or to the cylinder $\mathbb{S}^{1} \times \mathbb{R}$, or to the plane $\mathbb{R}^{2}$. If $I_{h c}^{*}$ is compact, then the flow on it is always complete and $I_{h c}^{*} \approx \mathbb{S}^{1} \times \mathbb{S}^{1}$.

(c) Under the hypothesis (b) the flow on $I_{h c}^{*}$ is conjugate to a linear flow on $\mathbb{S}^{1} \times \mathbb{S}^{1}$, on $\mathbb{S}^{1} \times \mathbb{R}$, or on $\mathbb{R}^{2}$.

The main result of this theorem is that the connected components of the invariant sets associated with the two independent first integrals in involution are generically submanifolds of the phase space, and if the flow on them is complete then they are diffeomorphic to a torus, a cylinder or a plane, where the flow is conjugate to a linear one.

Using the notation of Theorem 3 when a connected component $I_{h c}^{*}$ is diffeomorphic to a torus, either all orbits on this torus are periodic if the rotation number associated to this torus is rational, or they are quasi-periodic (i.e. every orbit is dense in the torus) if the rotation number associated to this torus is not rational.

We consider the autonomous differential system

$$
\dot{x}=f(x),
$$

where $f: U \rightarrow \mathbb{R}^{n}$ is $C^{2}, U$ is an open subset of $\mathbb{R}^{n}$ and the dot denotes the derivative respect to the time $t$. We write its general solution as $\phi\left(t, x_{0}\right)$ with $\phi\left(0, x_{0}\right)=x_{0} \in U$ and $t$ belonging to its maximal interval of definition. 
We say that $\phi\left(t, x_{0}\right)$ is $T$-periodic with $T>0$ if and only if $\phi\left(T, x_{0}\right)=x_{0}$ and $\phi\left(t, x_{0}\right) \neq x_{0}$ for $t \in(0, T)$. The periodic orbit associated to the periodic solution $\phi\left(t, x_{0}\right)$ is $\gamma=\left\{\phi\left(t, x_{0}\right), t \in[0, T]\right\}$. The variational equation associated to the $T$-periodic solution $\phi\left(t, x_{0}\right)$ is

$$
\dot{M}=\left(\left.\frac{\partial f(x)}{\partial x}\right|_{x=\phi\left(t, x_{0}\right)}\right) M,
$$

where $M$ is an $n \times n$ matrix. The monodromy matrix associated to the $T$-periodic solution $\phi\left(t, x_{0}\right)$ is the solution $M\left(T, x_{0}\right)$ of (13) satisfying that $M\left(0, x_{0}\right)$ is the identity matrix. The eigenvalues $\lambda$ of the monodromy matrix associated to the periodic solution $\phi\left(t, x_{0}\right)$ are called the multipliers of the periodic orbit.

For an autonomous differential system, one of the multipliers is always 1, and its corresponding eigenvector is tangent to the periodic orbit.

A periodic solution of an autonomous Hamiltonian system always has two multipliers equal to one. One multiplier is 1 because the Hamiltonian system is autonomous, and another one is 1 due to the existence of the first integral given by the Hamiltonian.

Theorem 4. If a Hamiltonian system with two degrees of freedom and Hamiltonian $H$ is Liouville-Arnol'd integrable, and $C$ is a second first integral such that the gradients of $H$ and $C$ are linearly independent at each point of a periodic orbit of the system, then all the multipliers of this periodic orbit are equal to 1.

Theorem 4 is due to Poincaré [16], section 36. It gives us a tool to study the non Liouville-Arnol'd integrability, independently of the class of differentiability of the second first integral. The main problem for applying this theorem is to find periodic orbits having multipliers different from 1.

5.2. Averaging Theory of First Order. Now we shall present the basic results from averaging theory that we need for proving the results of this paper.

The next theorem provides a first order approximation for the periodic solutions of a periodic differential system, for the proof see Theorems 11.5 and 11.6 of Verhulst [18].

Consider the differential equation

$$
\dot{\mathbf{x}}=\varepsilon F_{1}(t, \mathbf{x})+\varepsilon^{2} F_{2}(t, \mathbf{x}, \varepsilon), \quad \mathbf{x}(0)=\mathbf{x}_{0}
$$

with $\mathbf{x} \in D$, where $D$ is an open subset of $\mathbb{R}^{n}, t \geq 0$. Moreover we assume that both $F_{1}(t, \mathbf{x})$ and $F_{2}(t, \mathbf{x}, \varepsilon)$ are $T$-periodic in $t$. We also consider in $D$ the averaged differential equation

$$
\dot{\mathbf{y}}=\varepsilon f_{1}(\mathbf{y}), \quad \mathbf{y}(0)=\mathbf{x}_{0}
$$

where

$$
f_{1}(\mathbf{y})=\frac{1}{T} \int_{0}^{T} F_{1}(t, \mathbf{y}) d t
$$


Under certain conditions, equilibrium solutions of the averaged equation turn out to correspond with $T$-periodic solutions of equation (14).

Theorem 5. Consider the two initial value problems (14) and (15). Suppose:

(i) $F_{1}$, its Jacobian $\partial F_{1} / \partial x$, its Hessian $\partial^{2} F_{1} / \partial x^{2}, F_{2}$ and its Jacobian $\partial F_{2} / \partial x$ are defined, continuous and bounded by a constant independent of $\varepsilon$ in $[0, \infty) \times D$ and $\varepsilon \in\left(0, \varepsilon_{0}\right]$.

(ii) $F_{1}$ and $F_{2}$ are $T$-periodic in $t$ ( $T$ independent of $\varepsilon$ ).

Then the following statements hold.

(a) If $p$ is an equilibrium point of the averaged equation (15) and

$$
\left.\operatorname{det}\left(\frac{\partial f_{1}}{\partial \mathbf{y}}\right)\right|_{\mathbf{y}=p} \neq 0
$$

then there exists a $T$-periodic solution $\varphi(t, \varepsilon)$ of equation (14) such that $\varphi(0, \varepsilon) \rightarrow p$ as $\varepsilon \rightarrow 0$.

(b) The stability or instability of the limit cycle $\varphi(t, \varepsilon)$ is given by the stability or instability of the equilibrium point $p$ of the averaged system (15). In fact the singular point $p$ has the stability behavior of the Poincaré map associated to the limit cycle $\varphi(t, \varepsilon)$.

We point out the main facts in order to prove Theorem 5(b), for more details see Section 6.3 and 11.8 in [18].

5.3. Periodic orbits near equilibria. Consider the Hamiltonian system

$$
\dot{x}_{k}=H_{x_{n+k}}, \quad \dot{x}_{n+k}=-H_{x_{k}}, \quad k=1, \ldots, n,
$$

where $H_{x_{l}}$ denotes the partial derivative of the Hamiltonian $H\left(x_{1}, \ldots, x_{2 n}\right)$ with respect to the variable $x_{l}$. We can combine equations (16) in a single one writing

$$
\dot{x}=J H_{x}, \quad J=\left(\begin{array}{cc}
0 & I \\
-I & 0
\end{array}\right),
$$

where $H_{x}$ denotes the gradient of $H$, and where $I$ is the $n \times n$ identity matrix.

In 1973 Weinstein $[19,20]$ proved the following result.

Theorem 6 (Weinstein's Theorem). If the Hamiltonian $H$ is of class $\mathcal{C}^{2}$ near $x=0, H_{x}(0)=0$, and the Hessian $H_{x x}(0)$ is positive definite, then for any sufficiently small $\varepsilon$ the energy surface $H(x)=H(0)+\varepsilon^{2}$ contains at least $n$ periodic solutions of system (17) whose periods are close to those of the linear part of system (17) at the origin.

\section{ACKNOWLEDGMENTS}

The first author is partially supported by the grants MICINN/FEDER MTM 2008-03437, CIRIT 2009SGR 410 and ICREA Academia. This paper was written during a research visit of the second author to UAM, iztapalapa. 


\section{REFERENCES}

[1] Abraham R. and Marsden J.E., Foundations of Mechanics, Benjamin, Reading, Masachusets, 1978.

[2] Alfaro F., Llibre J. and Pérez-Chavela E., A class of galactic potentials: periodic orbits and integrability, preprint, 2011.

[3] Arnol'd V.I., Kozlov V. and Neishtadt A., Dynamical Systems III. Mathematical Aspects of Classical and Celestial Mechanics, Third Edition, Encyclopaedia of Mathematical Science, Springer, Berlin, 2006.

[4] Belmonte C., Boccaletti D. and Pucacco G., On the orbit structure of the logarithmic potential, The Astrophysical Journal 669 (2007), 202-217.

[5] Buică A. and Llibre J., Averaging methods for finding periodic orbits via Brouwer degree, Bull. Sci. Math. 128 (2004), 7-22.

[6] Holmes P., Proof of non-integrability for the Hénon-Heiles Hamiltonian near an exceptional integrable case, Physica D: Nonlinear Phenomena 5 (1982), 335-347.

[7] Jiménez-Lara L. and Llibre J., Periodic orbits and nonintegrability of generalized classical Yang-Mills Hamiltonian systems, J. Math. Physics 52 (2011), 032901 (9 pp).

[8] Jiménez-Lara L. and Llibre J., Periodic orbits and Hénon-Heiles systems, J. Phys. A: Math. Theor. 44 (2011), 205103 (14 pp).

[9] Kozlov V.V., Integrability and non-integrability in Hamiltonian mechanics, Russian Math. Surveys 38 No. 1 (1983), 1-76.

[10] Markus L. and Meyer, K.R., Generic Hamiltonian Dynamical Systems are neither integrable nor ergodic, Memoirs of the Amer. Math. Soc. 144, 1974.

[11] Meletlidou E. and Ichtiaroglou S., A criterion for non-integrability based on Poincaré's theorem, Physica D 71 (1994), 261-268.

[12] Meletlidou E. and Ichtiaroglou S., On the number of isolating integrals in perturbed Hamiltonian system with $n \geq 3$ degrees of freedom, J. Phys. A: Math. Gen. 27 (1994), 3919-3926.

[13] Meletlidou E., Stagika G. and Ichtiaroglou S., Non-integrability and structure of the resonance zones in a class of galactic potentials, Cel. Mech. and Dyn. Astron. 91 (2005), 323-335.

[14] Meyer K., Hall G. and Offin D., Introduction to Hamiltonian Dynamical Systems and the $N$-Body Problem, Second Edition, Applied Mathematical Sciences 90, Springer, 2009.

[15] Moser J., Stable and random motions in dynamical systems. With special emphasis on celestial mechanics, Annals of Mathematics Studies, No. 77, Princeton University Press, Princeton, N. J, 1973.

[16] Poincaré H., Les méthodes nouvelles de la mécanique céleste, Vol. I, Gauthier-Villars, Paris 1899.

[17] Pucacco G., Boccaletti D. and Belmonte C., Quantitative predictions with detuned normal forms, Celestial Mechanics and Dynamical Astronomy 102 (2008), 163-176.

[18] Verhulst F., Nonlinear Differential Equations and Dynamical Systems, Universitext, Springer, 1991

[19] Weinstein A., Lagrangian submanifolds and Hamiltonian systems, Ann. Math. 98 (1973), 377-410.

[20] Weinstein A, Normal modes for nonlinear Hamiltonian systems, Inventiones Math. 20 (1973), 47-57. 